YanYan Li · Louis Nirenberg

\title{
A geometric problem and the Hopf Lemma. I
}

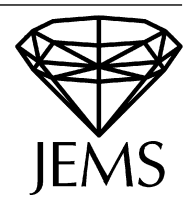

\author{
Dedicated to Antonio Ambrosetti on his 60th birthday
}

Received July 14, 2005

\begin{abstract}
A classical result of A. D. Alexandrov states that a connected compact smooth $n$-dimensional manifold without boundary, embedded in $\mathbb{R}^{n+1}$, and such that its mean curvature is constant, is a sphere. Here we study the problem of symmetry of $M$ in a hyperplane $X_{n+1}=$ const in case $M$ satisfies: for any two points $\left(X^{\prime}, X_{n+1}\right),\left(X^{\prime}, \widehat{X}_{n+1}\right)$ on $M$, with $X_{n+1}>\widehat{X}_{n+1}$, the mean curvature at the first is not greater than that at the second. Symmetry need not always hold, but in this paper, we establish it under some additional condition for $n=1$. Some variations of the Hopf Lemma are also presented. Part II [Y.Y. Li and L. Nirenberg, Chinese Ann. Math. Ser. B 27 (2006), 193-218] deals with corresponding higher dimensional problems. Several open problems for higher dimensions are described in this paper as well.
\end{abstract}

\section{Introduction}

The problem we consider starts with the following classical result of A. D. Aleksandrov [1]:

Theorem 1.1 ([1]). Let $M$ be a compact smooth hypersurface, without boundary, embedded in $\mathbb{R}^{n+1}$ with the property that the mean curvature (average of principle curvatures, using interior normal) is identically constant. Then $M$ is a sphere.

If $M$ is immersed instead of embedded, the conclusion of the theorem may fail, even in dimension $n=2$. Indeed, in 1986, Wente [6] constructed a counter-example in case $M$ is an immersed torus, with self-intersection, in $\mathbb{R}^{3}$. A. Ros [5] in 1987 extended Theorem 1.1 from mean curvature to the elementary symmetric functions of the principal curvatures of $M$. In 1997 YanYan Li [2] gave some far reaching generalizations including very general symmetric functions of the principal curvatures of $M$. But here we just mention one of the results—still for the mean curvature.

Y.Y. Li was partially supported by NSF grant DMS-0401118. L. Nirenberg thanks the Pacific Institute of Mathematical Sciences for support. This is a report of a talk given at the January 2005 conference in Rome in celebration of A. Ambrosetti's birthday.

Y.Y. Li: Department of Mathematics, Rutgers University, 110 Frelinghuysen Road, Piscataway, NJ 08854, USA; e-mail: yyli@ math.rutgers.edu

L. Nirenberg: Courant Institute, 251 Mercer Street, New York, NY 10012, USA;

e-mail: nirenl@cims.nyw.edu 
Theorem 1.2 ([2]). Let $M$ be a compact smooth hypersurface without boundary embedded in $\mathbb{R}^{n+1}$. Let $K$ be a $C^{1}$ function in $\mathbb{R}^{n+1}$ satisfying

$$
\frac{\partial K}{\partial x_{n+1}} \leq 0 \text {. }
$$

Suppose that at each point $x$ of $M$ the mean curvature $H(x)$ equals $K(x)$. Then $M$ is symmetric about some hyperplane

$$
x_{n+1}=\lambda_{0} .
$$

Li then proposed that we consider the more general question in which the condition $H(x)=K(x)$ with $K$ satisfying (1) is replaced by the weaker, more natural, condition: Whenever $\left(x^{\prime}, a\right)$ and $\left(x^{\prime}, b\right), a<b$, lie on $M$ (here $\left.x^{\prime}=\left(x_{1}, \cdot, x_{n}\right)\right)$ then

$$
H\left(x^{\prime}, b\right) \leq H\left(x^{\prime}, a\right) .
$$

Question 1.1. Is it true that $M$ is then symmetric about some hyperplane $x_{n+1}=\lambda_{0}$ ?

This paper-here we consider only one-dimensional problems-and its sequel are concerned with this question.

First we recall Aleksandrov's argument. It introduces the, now familiar, method of moving planes, and the proof relies on the strong maximum principle and the Hopf Lemma for second order elliptic equations. Here it is: $M$ is the boundary of an open set $U$ in $\mathbb{R}^{n+1}$. For $\lambda$ less than, but close to, $\max _{M} x_{n+1}$, take the part $S_{\lambda}$ of $M$ lying above $\lambda$ (i.e. with $x_{n+1}>\lambda$ ) and reflect it in the plane $x_{n+1}=\lambda$. The reflected piece of surface, $S_{\lambda}^{\prime}$, lies in $\bar{U}$. Decrease $\lambda$ and continue to reflect $S_{\lambda}$ so that $S_{\lambda}^{\prime}$ continues to lie in $\bar{U}$. There will be a first value $\lambda_{0}$ of $\lambda$ such that one of the two things happen:

(i) $S_{\lambda_{0}}^{\prime}$ touches $M$ at some point $\left(x_{0}^{\prime}, a_{0}\right)$ with $a_{0}<\lambda$, and the line $\left\{\left(x_{0}^{\prime}, x_{n+1}\right) \mid x_{n+1} \in\right.$ $\left.\mathbb{R}^{n+1}\right\}$ is transversal to $M$ at $\left(x_{0}^{\prime}, a_{0}\right)$, or

(ii) at some point $P$ on $x_{n+1}=\lambda_{0}$ the hypersurfaces $S_{\lambda_{0}}$ and $M$ are tangent to each other.

Note that both things may happen at the same $\lambda_{0}$.

In Case (i) we may describe $M$ and $S_{\lambda_{0}}^{\prime}$ near $\left(x_{0}^{\prime}, a_{0}\right)$ as graphs of smooth functions $v\left(x^{\prime}\right), u\left(x^{\prime}\right)$ with

$$
v\left(x^{\prime}\right) \leq u\left(x^{\prime}\right) \quad \text { and } \quad v\left(x_{0}^{\prime}\right)=u\left(x_{0}^{\prime}\right) .
$$

Both functions satisfy, near $x_{0}^{\prime}$, the nonlinear elliptic equation expressing the fact that the mean curvature is the constant $H$,

$$
H[u]:=\nabla\left(\frac{\nabla u}{\sqrt{1+|\nabla u|^{2}}}\right)=H .
$$

But by the strong maximum principle it follows from 4 that $u \equiv v$ near $x_{0}^{\prime}$. Applying this argument at other points on $M$ shows that

$$
S_{\lambda_{0}}^{\prime}=\left\{x \in M \mid x_{n+1}<\lambda_{0}\right\}
$$

This is the desired symmetry. 


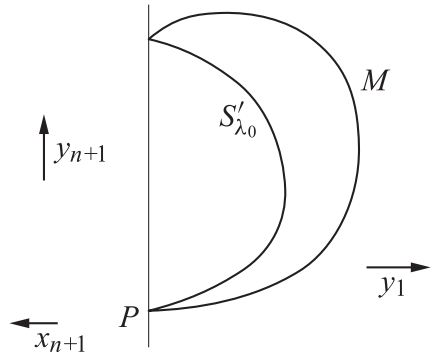

Fig. 1

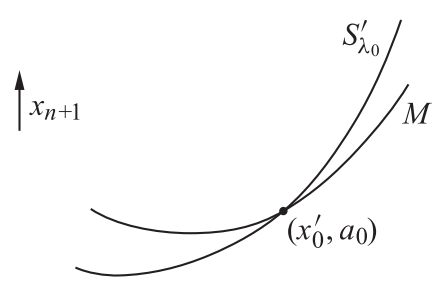

Fig. 2

In Case (ii) we turn the picture around (see Fig. 1). In these coordinates, call them $y$, with $y_{1}=P_{1}-x_{n+1}$, we may represent $S_{\lambda_{0}}^{\prime}$, near $P$, as the graph of a function $u\left(y^{\prime}\right)$, and the part of $M$ lying in $y_{1}>0$ as the graph of some $v\left(y^{\prime}\right)$. We have, with some abuse of notation,

$$
u\left(y^{\prime}\right) \geq v\left(y^{\prime}\right), \quad u(P)=v(P), \quad \nabla u(P)=\nabla v(P) .
$$

As before, $u$ and $v$ satisfy the same elliptic equation in $y_{1}>0$, near the origin. By the Hopf Lemma, $u \equiv v$ near the origin. Then, using the strong maximum principle we extend this fact globally, to conclude the desired symmetry.

In [2] Li uses the moving plane method but makes essential use of the fact that the function $K$ is locally Lipschitz in $\mathbb{R}^{n+1}$.

What happens if, following Aleksandrov, we try to use moving planes for the problem where $H\left(x^{\prime}, b\right) \leq H\left(x^{\prime}, a\right)$ for $b>a$ ? We are led again to the two cases (i) and (ii) above. Case (i) is easily handled (see Fig. 2). Again we have two functions $u\left(x^{\prime}\right) \geq v\left(x^{\prime}\right)$. But now $H[u] \leq H[v]$. We may still use the strong maximum principle and infer that $u \equiv v$.

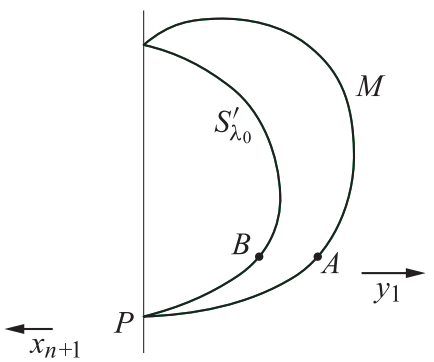

Fig. 3

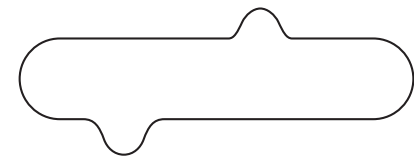

Fig. 4

The trouble arises in Case (ii). We redraw Fig. 1 as shown in Fig. 3. We may represent $S_{\lambda_{0}}^{\prime}$ and $M$ in $y_{1}>0$ by $u\left(y^{\prime}\right)$ and $v\left(y^{\prime}\right)$, with $u \geq v$. However, the condition that the mean curvature of $S_{\lambda_{0}}^{\prime}$ at $B$ is $\leq$ that at $A$, compares the mean curvature of $u$ and $v$ but at different points, $\left(y_{1}, y^{\prime \prime}\right)$ and $\left(\bar{y}_{1}, y^{\prime \prime}\right)$ where $u\left(y_{1}, y^{\prime \prime}\right)=v\left(\bar{y}_{1}, y^{\prime \prime}\right)$, with $y_{1} \leq \bar{y}_{1}$.

Thus we are led to looking for a more general form of the Hopf Lemma. 
Before stating some results, we point out that the answer to Question 1.1 is no in general, even for a closed curve with interior convex in the $x_{2}$-direction, as in Fig. 4 . Here the ends are symmetric to each other, the bottom bump is symmetric, as is the top bump.

Even if $M$ is not necessarily symmetric, would the inequality on the mean curvature imply that equality holds in the following sense:

Question 1.2. Is it true that (3) implies that for any $A, B \in M$ with $A_{n+1}<B_{n+1}$, we must have $H(A)=H(B)$ ?

In Section 6 we give a counterexample. However, we do not know the answer to

Question 1.3. Is the answer to Question 1.2 yes in case we consider $A, B \in M$ such that for all $0<t<1, t A+(1-t) B$ lies inside $M$ ?

In Part II, [3], we present our results on Question 1.1. We assume that the (embedded) hypersurface is smooth and satisfies

Condition S. $M$ stays on one side of any hyperplane parallel to the $x_{n+1}$-axis that is tangent to $M$.

We believe that this should suffice to prove symmetry. However our proof requires a further condition:

Condition T. Any line parallel to the $x_{n+1}$-axis that is tangent to $M$ has contact of finite order.

Condition T automatically holds in case $M$ is analytic; while Condition S automatically holds in case $M$ is convex.

A weaker version of our main result is

Theorem 1.3 ([3]). Let $M$ be a smooth compact embedded hypersurface in $\mathbb{R}^{n+1}$ satisfying

$$
H\left(x^{\prime}, x_{n+1}\right) \leq H\left(x^{\prime}, \tilde{x}_{n+1}\right)
$$

for any two points $\left(x^{\prime}, x_{n+1}\right),\left(x^{\prime}, \tilde{x}_{n+1}\right) \in M$ satisfying $x_{n+1} \geq \tilde{x}_{n+1}$. Then, if Condition $T$ holds and $M$ is locally convex near any point where the tangent plane is parallel to the $x_{n+1}$-axis, $M$ must be symmetric with respect to some hyperplane $x_{n+1}=$ const.

In this paper we restrict ourselves to curves. The main result in Part I is

Theorem 1.4. Let $M$ be a closed $C^{2}$ embedded curve in the plane satisfying Condition $S$. Assume that whenever $\left(x_{1}, a\right),\left(x_{1}, b\right)$, with $a<b$, lie on the curve $M$, then

$$
\text { curvature of } M \text { at }\left(x_{1}, b\right) \leq \text { curvature of } M \text { at }\left(x_{1}, a\right) \text {. }
$$

Then $M$ is symmetric about some line $x_{2}=\lambda_{0}$.

Remark 1.1. In Theorem 1.4, we do not assume Condition $\mathrm{T}$.

The theorem is proved in Section 3. In Part II, in addition to mean curvature, we also extend Theorem 1.3 to other symmetric functions of the principal curvatures. A number of open problems are also presented there. In Section 7 of this Part I we also mention several which are local in nature. 


\section{One-dimensional model problems}

We first looked at some very simple one-dimensional model problems which seemed to us to be of interest. Here is one of them.

Theorem 2.1. Let $u \geq v$ be positive $C^{2}$ functions on $(0, b)$, which are also in $C^{1}([0, b])$. Assume that

$$
u(0)=\dot{u}(0)=0,
$$

and

$$
\text { either } \dot{u}>0 \text { on }(0, b) \quad \text { or } \quad \dot{v}>0 \text { on }(0, b) .
$$

Our main hypothesis is:

$$
\text { whenever } u(t)=v(s) \text { for } 0<t<s<b \text { we have } \ddot{u}(t) \leq v^{\prime \prime}(s)
$$

(here $\left.\cdot=d / d t,{ }^{\prime}=d / d s\right)$. Conclusion:

$$
u \equiv v \quad \text { on }[0, b] .
$$

Remark 2.1. This is a kind of extension of the Hopf Lemma, for if in place of (7) we assumed

$$
\ddot{u}(t) \leq \ddot{v}(t) \quad \text { on }(0, b)
$$

the result would simply follow from the Hopf Lemma.

Remark 2.2. If we replace (6) by both $\dot{u} \geq 0$ and $\dot{v} \geq 0$ on $(0, b)$, the conclusion of Theorem 2.1 may fail. See the following example.

Example 2.1. Let $u \in C^{\infty}([0,2])$ satisfy

$$
\begin{gathered}
u(t)= \begin{cases}t^{3}, & 0 \leq t \leq 1 / 3 \\
1+(t-1)^{3}, & 2 / 3 \leq t \leq 2,\end{cases} \\
\dot{u}>0 \quad \text { in }(0,1)
\end{gathered}
$$

and let

$$
v(t)=w(t)= \begin{cases}u(t), & 0 \leq t \leq 1 \\ 1, & 1<t \leq 2\end{cases}
$$

as in Fig. 5.

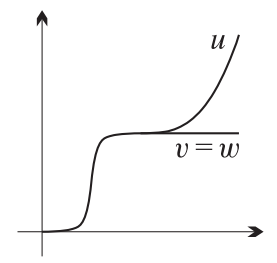

Fig. 5

Before proving Theorem 2.1, we give a few lemmas. Some of these are not really used in the proof of Theorem 2.1, but seem of interest.

First, a variation of the strong maximum principle. 
Lemma 2.1. Let $u \geq v$ be two $C^{2}$ functions on $(0, b)$. Assume (7) and

$$
\max \{\dot{u}, \dot{v}\}>0 \quad \text { on }(0, b) .
$$

Then either

$$
u>v \quad \text { on }(0, b),
$$

or

$$
u \equiv v \quad \text { on }(0, b)
$$

Remark 2.3. If we replace $(9)$ by $\dot{u}, \dot{v} \geq 0$ on $(0, b)$, the conclusion of the lemma may fail. See Example 2.1 above.

Proof of Lemma 2.1. Suppose $u(c)=v(c)$ for some $0<c<b$. Then, by 9],

$$
\dot{u}(c)=\dot{v}(c)>0 .
$$

By the implicit function theorem, for $s$ close to $c$, there is a $C^{2}$ function $t(s)$ such that

$$
u(t(s))=v(s) .
$$

For $s$ close to $c$, set

$$
g(s)=s-t(s),
$$

so $g \geq 0$. Differentiating (11) we find

$$
\dot{u}(t(s)) t^{\prime}=v^{\prime}(s), \quad \ddot{u} t^{\prime 2}+\dot{u} t^{\prime \prime}=v^{\prime \prime} .
$$

In terms of $g$ the last equation becomes, by (7),

$$
0 \geq \ddot{u}(t(s))-v^{\prime \prime}(s)=\dot{u} g^{\prime \prime}-g^{\prime}\left(g^{\prime}-2\right) \ddot{u} .
$$

Now if $u(s)=v(s)$ for some $s>0$ then $g$ vanishes there. The strong maximum principle applied to (12) implies that $v \equiv u$ in a neighborhood of $c$. By the same argument, $u \equiv v$ in a larger neighborhood, and (10) then follows. Lemma 2.1] is established.

Lemma 2.2. Let $u$ and $w$ be positive $C_{\text {loc }}^{1,1}$ functions on $(0, c)$, belonging to $C^{1}([0, c))$, and satisfying, for some $f \in L_{\mathrm{loc}}^{\infty}(0, \infty)$,

$$
\ddot{u}=f(u), \quad \ddot{w}=f(w) \quad \text { in }(0, c),
$$

and

$$
u(0)=w(0)=0, \quad \dot{u}(0)=\dot{w}(0), \quad \dot{u}>0 \quad \text { on }(0, c) .
$$

Then

$$
u \equiv w \quad \text { on }(0, c) .
$$

Remark 2.4. We do not assume $\dot{w}>0$. On the other hand, if we replace $\dot{u}>0$ by $\dot{u} \geq 0$ in the hypotheses, the conclusion may fail. See Example 2.1.

Here is another simple uniqueness result; it could be taught in a beginning course on ordinary differential equations. 
Lemma 2.3. Let $u$ and $w$ be positive $C_{\text {loc }}^{1,1}$ functions in $(0, c)$, belonging to $C^{1}([0, c))$, and both satisfying

$$
\ddot{u}=f(u),
$$

and

$$
u(0)=w(0)=0, \quad \dot{u}(0)=\dot{w}(0) .
$$

Assume that $f(\varrho)$ is locally Lipschitz for $\varrho>0$ (not necessarily for $\varrho \geq 0$ ). Then

$$
u \equiv w .
$$

Note that we assume neither $\dot{u} \geq 0$ nor $\dot{w} \geq 0$.

Proof of Lemma 2.3 The proof is by obtaining an "explicit" expression for $u(t)$. Multiply (13) by $2 \dot{u}(t)$ and integrate from some $t_{0}>0$ to some $t>t_{0}$. We find

$$
\dot{u}(t)^{2}-\dot{u}\left(t_{0}\right)^{2}=F(u(t))-F\left(u\left(t_{0}\right)\right) .
$$

Here $F(\varrho)$ is such that

$$
\frac{d F}{d \varrho}=2 f(\varrho) \quad \text { for } \varrho>0 .
$$

Letting $t_{0} \rightarrow 0$ we see that $F(\varrho)$ has a limit as $\varrho \rightarrow 0$, which we may take to be $\dot{u}(0)^{2}$. Thus, letting $t_{0} \rightarrow 0$ we find

$$
\dot{u}(t)^{2}=F(u(t)) .
$$

Claim. On $(0, c / 2), \dot{u}>0$.

For if not, if $\dot{u}\left(t_{1}\right)=0$ for some $0<t_{1}<c / 2$, then the local Lipschitz property of $f$ for $\varrho>0$ would imply that the function $u$ is symmetric about $t_{1}$. But then it would have to vanish at $2 t_{1}-$ where $u$ is positive.

Consequently, from (14), we find that $F(\varrho)>0$ for $0<\varrho<u(c / 4)$, and

$$
\dot{u}(t)=\sqrt{F(u(t))}
$$

or

$$
\frac{\dot{u}}{\sqrt{F(u)}}=1 .
$$

If, on $\varrho>0, G(\varrho)$ is such that

$$
G_{\varrho}=\frac{1}{\sqrt{F(\varrho)}},
$$

we find from $(15)$ that

$$
\frac{d}{d t} G(u)=1
$$

Integrating from $t_{0}>0$ to $t>t_{0}$ we obtain

$$
G(u(t))-G\left(u\left(t_{0}\right)\right)=t-t_{0} .
$$

Letting again $t_{0} \rightarrow 0$ we see that $G$ has a limit at $\varrho=0$, which we may take to be zero. Thus

$$
G(u(t))=t .
$$


So $u(t)$ is uniquely determined on $(0, c / 2)$, since $G_{\varrho}>0$ for $\varrho>0$. Then, by the local Lipschitz property of $f(\varrho)$ on $\varrho>0$, it follows that $u$ is unique on $(0, c)$. Lemma 2.3 is proved.

Proof of Lemma 2.2. Follow the proof of Lemma 2.3 until (14. Similarly we also have

$$
\dot{w}(t)^{2}=F(w(t)) \quad \text { on }(0, c) .
$$

Let $0<b<c$ be any number satisfying

$$
\max _{[0, b]} w<\sup _{[0, c)} u=\lim _{t \rightarrow c^{-}} u(t)
$$

We will prove that

$$
u \equiv w \quad \text { on }[0, b]
$$

By (14), (16) and the fact that $\dot{u}>0$ on $(0, c)$, we know that

$$
\dot{w}(t)^{2}=F(w(t))>0, \quad \forall 0<t<b .
$$

Since $w(0)=0$ and $w>0$ on $(0, b)$, we have, in view of the above, $\dot{w}>0$ on $(0, b)$. Proceeding as in the proof of Lemma 2.3 , we arrive at

$$
G(u(t))=G(w(t))=t, \quad 0<t<b .
$$

But $G_{\varrho}>0$ and $G(0)=0$, and we obtain $(18)$ as before.

Arguing in the same way we see that $u \equiv w$ on an interval $\left(0, b^{\prime}\right), b^{\prime}>b$, and then on $(0, c)$.

The following lemma can be viewed as a variation of the maximum principle.

Lemma 2.4. Let $u, v \in C^{2}((0, b)) \cap C^{0}([0, b])$ satisfy

$$
\begin{gathered}
v(0) \leq u(0), \quad v<u(b) \quad \text { on }(0, b), \\
\text { either } \dot{u}>0 \text { on }(0, b), \quad \text { or } \quad \dot{v}>0 \text { whenever } u(0)<v<u(b),
\end{gathered}
$$

and (7). Then

$$
u \geq v \quad \text { on }[0, b] .
$$

Remark 2.5. If we change (19) to $\dot{u} \geq 0$ and $\dot{v} \geq 0$, the conclusion of Lemma 2.4 may fail. See the example below.

Example 2.2. Let $u \in C^{\infty}([0,4])$ satisfy

$$
u(t)=\left\{\begin{array}{ll}
1, & 0 \leq t \leq 2 \\
1+(t-2)^{3}, & 2<t \leq 7 / 3, \\
2, & 8 / 3<t \leq 3, \\
2+(t-3)^{3}, & 3<t \leq 4
\end{array} \quad \dot{u}>0 \quad \text { in }(7 / 3,8 / 3)\right.
$$




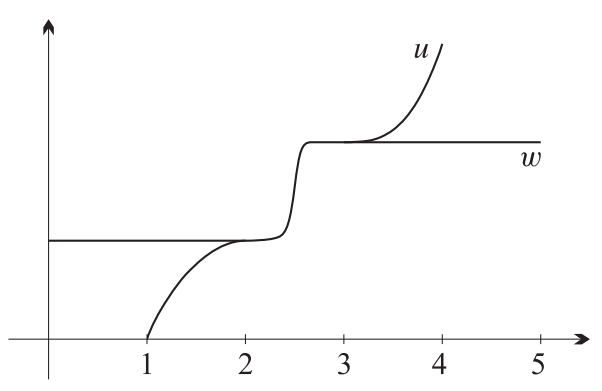

Fig. 6

and let $w \in C^{\infty}([1,5])$, with $w^{\prime}$ nonnegative, satisfy $w(1)=0$,

$$
w(t)= \begin{cases}u(t), & 2 \leq t \leq 3 \\ 2, & 3<t \leq 5\end{cases}
$$

Then let

$$
v(t)=w(t+1), \quad 0 \leq t \leq 4 .
$$

See Fig. 6.

Proof of Lemma 2.4 Shift $v$ far to the right and then slide it to the left until its graph first touches that of $u$. If the touching occurs at $(c, u(c))$ for some $0<c<b$, then $u^{\prime}(c)>0$, and therefore, by Lemma 2.1 $u$ and the shift of $v$ must coincide near $c$. Again by that lemma, the set of points where $u \equiv v$ is open. Since it is also closed, we conclude that the shift of $v$ is $v$ itself and $u \equiv v$. Otherwise, we can slide the shift of $v$ all the way to the origin and we conclude $u \geq v$ on $(0, b)$.

Proof of Theorem 2.1. (a) We first assume that $\dot{u}>0$ on $(0, b)$. Because of Lemma 2.1. we may suppose that

$$
u(t)>v(t) \quad \text { for } t>0,
$$

and we will derive a contradiction.

Our proof makes use of the fact that $u$ satisfies some differential equation. Namely, since $\dot{u}(t)>0$ for $t>0$, we see that for $u>0, t$ is a $C^{2}$ function of $u$. It follows that we may write

$$
\ddot{u}=f(u),
$$

with $f$ some unknown function which is however continuous in $u$ on $[0, u(b)]$. The main hypothesis (7) is then equivalent to the following for $v$ :

$$
v^{\prime \prime}(s) \geq f(v(s)) .
$$

Thus $v$ is a subsolution of 20] while $u \geq v$ is a solution.

Consequently, there is a solution $w$ of (20) lying between $u$ and $v$, with

$$
w(0)=0, \quad w(c)=v(c) \quad \text { for some fixed } c \text { in }(0, \bar{b}) .
$$


If $\ddot{u}$ is locally Lipschitz on $(0, b)$, then $f$ is locally Lipschitz continuous in $(0, u(b)]$ and we can see this by first, for small positive $\epsilon$, finding a solution $w_{\epsilon}$ between $u$ and $v$ on $[\epsilon, c]$ with

$$
w(\epsilon)=v(\epsilon), \quad w(c)=v(c) .
$$

Because of the locally Lipschitz property of $f$, the usual argument by monotone iterations yields $w_{\epsilon}$. Letting $\epsilon \rightarrow 0$ one easily obtains a solution $w$ satisfying 20] with

$$
v(t) \leq w(t) \leq u(t) .
$$

J. Mawhin pointed out to us that the approximation by $w_{\epsilon}$ is not necessary. That there is a solution between $u$ and $v$, even for merely continuous $f$, is known (see [4]).

We now have two positive solutions of $(20)$ in $(0, c), u$ and $w$, with $u(0)=\dot{u}(0)=$ $w(0)=\dot{w}(0)=0$. By Lemma 2.2 $u \equiv w$ on $(0, c)$, violating $u(c)>v(c)$. Impossible.

(b) We now assume that $\dot{v}>0$ on $(0, b)$. Let $0<a<b$ be any number satisfying

$$
\max _{[0, a]} u<\sup _{[0, b)} v=\lim _{t \rightarrow b^{-}} v(t) .
$$

We will prove that

$$
u \equiv v \quad \text { on }[0, a] .
$$

It is easy to see that this would imply $u \equiv v$ on $[0, b]$.

Since $\dot{v}>0$ on $(0, b)$ it follows, in view of 22, , that for every $t \in(0, a)$ there is a $C^{2}$ function $s(t)$ such that

$$
u(t)=v(s(t)), \quad 0<t<a .
$$

Set

$$
g(t)=s(t)-t, \quad 0<t<a,
$$

so $g \geq 0$. Differentiating 24] we find, still using the notation $\cdot=d / d t,{ }^{\prime}=d / d s$,

$$
\dot{u}(t)=v^{\prime}(s(t)) \dot{s}(t), \quad \ddot{u}=v^{\prime \prime} \dot{s}^{2}+v^{\prime} \ddot{s} .
$$

In terms of $g$ the last equation becomes, by (7),

$$
0 \geq \ddot{u}(t)-v^{\prime \prime}(s(t)) \geq v^{\prime} \ddot{g}+\dot{g}(\dot{g}+2) v^{\prime \prime} .
$$

If $u(t)=v(t)$ for some $0<t<a$, then $g$ vanishes there and, as before, $g \equiv 0$ on $(0, a)$, which in turn implies 23.

Thus we may assume that

$$
u>v \quad \text { on }(0, a),
$$

and we will derive a contradiction.

Since $v^{\prime}>0$ on $(0, b)$, we may, as before, write

$$
v^{\prime \prime}=f(v) \quad \text { on }(0, b)
$$

where $f$ is some unknown continuous function on $\left[0, \lim _{s \rightarrow b^{-}} v(s)\right)$. By our main hypothesis (7), in view of (22),

$$
\ddot{u} \leq f(u) \quad \text { on }(0, a) \text {. }
$$


As before there exists a solution $w$ of 25 lying between $u$ and $w$, with

$$
w(0)=0, \quad w(a)=v(a) .
$$

By Lemma 2.2 $u \equiv w$ on $[0, a]$, violating $u(a)>v(a)$. Theorem 2.1 is established.

\section{Proof of Theorem 1.4}

We first give the main lemma for the proof of Theorem 1.4

Lemma 3.1. Let, for some $b>0, u, v \in C^{2}((0, b)) \cap C^{1}([0, b])$ satisfy

$$
u(t) \geq v(t)>0 \quad \text { for } 0<t \leq b,
$$

either $\dot{u}(t)>0, \dot{v}(t) \geq 0$ for $0<t \leq b, \quad$ or $\quad \dot{u}(t) \geq 0, \dot{v}(t)>0$ for $0<t \leq b$,

and

$$
u(0)=\dot{u}(0)=0
$$

Assume

$$
\text { whenever } u(t)=v(s) \text { for } 0<t<s<b \quad \text { we have } \quad \frac{\ddot{u}(t)}{\left(1+\dot{u}^{2}\right)^{3 / 2}} \leq \frac{v^{\prime \prime}(s)}{\left(1+v^{\prime 2}\right)^{3 / 2}} \text {. }
$$

Then

$$
u \equiv v \quad \text { on }[0, b]
$$

Proof. We will only prove it under the assumption " $\dot{u}(t)>0, \dot{v}(t) \geq 0$ for $0<t \leq b$ ". The changes needed when assuming instead " $\dot{u}(t) \geq 0, \dot{v}(t)>0$ for $0<t \leq b$ " are similar to those in the proof of Theorem 2.1. We start as in the proof of Theorem 2.1. $u$ satisfies an equation of the form

$$
\frac{\ddot{u}}{\left(1+\dot{u}^{2}\right)^{3 / 2}}=f(u)
$$

with some unknown function $f$ which is however continuous on $(0, u(b)]$.

Our condition 26 means that

$$
\frac{\ddot{v}}{\left(1+\dot{v}^{2}\right)^{3 / 2}} \geq f(v)
$$

Multiplying (28) by $\dot{u}$, we find that

$$
-\frac{d}{d t}\left(\frac{1}{\left(1+\dot{u}^{2}\right)^{1 / 2}}\right)=\frac{d}{d t} F(u)
$$

where $F$ is such that $F_{u}=f(u)$. Integrating this from $t_{0}$ to $t, t_{0}>0$, we find

$$
\left(\frac{1}{\left(1+\dot{u}\left(t_{0}\right)^{2}\right)^{1 / 2}}\right)^{1 / 2}-\left(\frac{1}{\left(1+\dot{u}(t)^{2}\right)^{1 / 2}}\right)^{1 / 2}=F(u(t))-F\left(u\left(t_{0}\right)\right) .
$$


Letting $t_{0} \rightarrow 0$ we see that $F(u)$ has a limit at $u=0$, which we may take to be zero. Thus

$$
\left(\frac{1}{1+\dot{u}^{2}}\right)^{1 / 2}=1-F(u)
$$

so that

$$
\dot{u}=\left[\frac{1}{(1-F(u))^{2}}-1\right]^{1 / 2}
$$

Next, multiplying 30 by $\dot{v} \geq 0$ we obtain

$$
-\frac{d}{d t}\left(\frac{1}{1+\dot{v}^{2}}\right)^{1 / 2} \geq \frac{d}{d t} F(v) \quad \text { for } 0<t_{0}<t .
$$

Since $F(0)=0$, we find, on integrating,

$$
1-\left(\frac{1}{1+\dot{v}^{2}}\right)^{1 / 2} \geq F(v(t))
$$

Thus, since $\dot{v} \geq 0$,

$$
\dot{v} \geq\left[\frac{1}{(1-F(v(t)))^{2}}-1\right]^{1 / 2} .
$$

But 30 and 31 imply that

$$
\text { whenever } u(t)=v(s) \text { for } t<s, \quad \text { then } \quad \dot{u}(t) \leq v^{\prime}(s) .
$$

Since $\dot{u}>0$ for $t>0$, by the implicit function theorem, there is a $C^{1}$ function $t \leq s$ such that

$$
u(t(s))=v(s) .
$$

Thus if $g=s-t \geq 0$, we have by differentiating,

$$
\dot{u}(t)\left(1-g^{\prime}\right)=v^{\prime}(s) .
$$

From 32 it follows that

$$
\dot{u} g^{\prime}=\dot{u}-v^{\prime} \leq 0,
$$

i.e. $g^{\prime} \leq 0$. Since $g(0)=0$ and $g \geq 0$, it follows that $g \equiv 0$-which implies 27).

Proof of Theorem 1.4 Condition S implies that there are just two lines parallel to the $x_{2}$ axis which are tangent to $M$. We apply the moving plane method as described in Section 1 except that we define $\lambda_{0}$ to be the first value of $\lambda$, as we decrease it, such that for any $\overline{\lambda<\lambda_{0}, S_{\lambda}^{\prime}}$ does not lie in $\bar{U}$. We then obtain Cases (i) and (ii) as in Section 1.

If Case (i) happens, then it can be treated as described there, but with some difference: flat vertical segments may occur, though we still obtain symmetry. See Fig. 7.

Now we look at Case (ii). There is a common tangency point of $S_{\lambda_{0}}^{\prime}$ and $M$ such that if we rotate the figure it looks as in Fig. 8 or Fig. 9, with coordinates $t$ and $y$, and, due to Condition $\mathrm{S}$, the curves lie above the $t$-axis. 


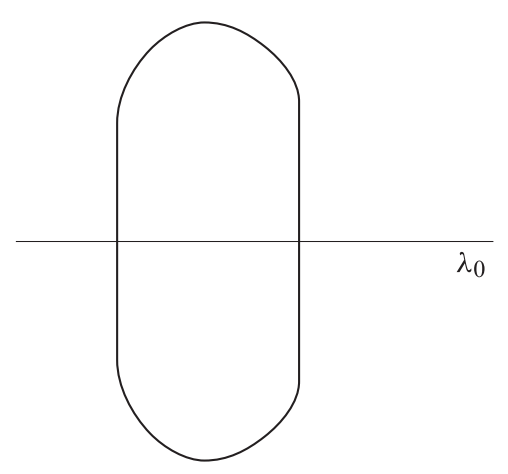

Fig. 7

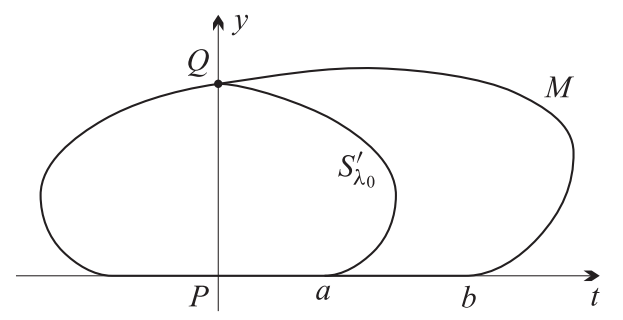

Fig. 8

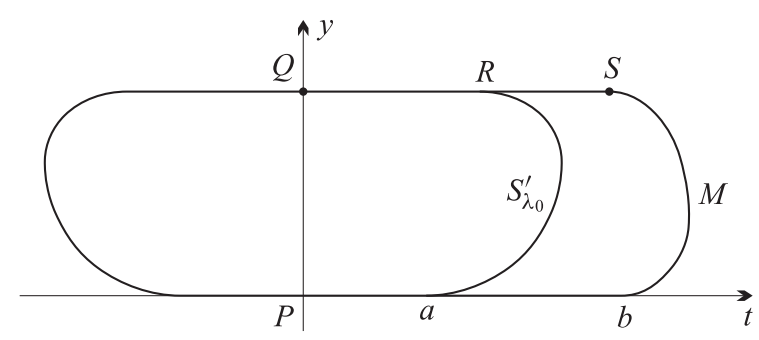

Fig. 9

Again, we are allowing $M$ to have some flat segment. Take as origin the point $P$ as shown. Let $[0, a]$ be the largest interval for the flat segment of $S_{\lambda_{0}}^{\prime}$ and let $[0, b]$ be the largest interval for the flat segment of $M$ as shown. By Condition S, $S_{\lambda_{0}}^{\prime}$ does not intersect the $t$-axis after $a$ and $M$ does not intersect the $t$-axis after $b$. If $a=b$, then we represent, for $t>a$ but close to $a, S_{\lambda_{0}}^{\prime}$ by $y=u(t)$ and $M$ by $y=v(t)$. By Condition $\mathrm{S}$, we know that $\dot{u}, \dot{v}>0$ for $t>a$ and $t$ close to $a$. Lemma 3.1 yields $u \equiv v$ near $t=a$. The situation is now reduced to Case (i) and the symmetry of $M$ follows.

If $a<b$, then Case (i) cannot occur. Thus, by the definition of $\lambda_{0}$, both $S_{\lambda_{0}}^{\prime}$ and $M$ must have a horizontal tangent line at $Q$. Let, as shown in Fig. 9, $[Q, R]$ be the largest flat segment on the top part of $S_{\lambda_{0}}^{\prime}$ and $[Q, S]$ be the largest flat segment on the top part 
of $M$. Then applying Lemma 3.1 as above, we see that $R$ and $S$ must be different. Recall that Case (i) does not happen. It is then clear that for all $\lambda$ close to $\lambda_{0}, S_{\lambda}^{\prime}$ still lies in $\bar{U}$, contradicting the definition of $\lambda_{0}$. Theorem 1.4 is established.

The remaining sections take up some further one-dimensional model problems.

\section{More results}

Lemma 4.1. Let $u \in C^{2}((a, b)) \cap C^{1}([a, b])$ satisfy

$$
\dot{u}(a)=0
$$

and

$$
\dot{u}>0 \quad \text { on }(a, b) \text {. }
$$

Let $v \in C^{2}((\alpha, \beta)) \cap C^{1}([\alpha, \beta])$ satisfy

$$
v(\alpha) \leq u(b), \quad v(\beta)=u(a), \quad \text { and } \quad v(\beta)<v(s)<v(\alpha), \quad \forall \alpha<s<\beta,
$$

and

$$
\dot{v}(\alpha)=0 \text {. }
$$

See for example Fig. 10.
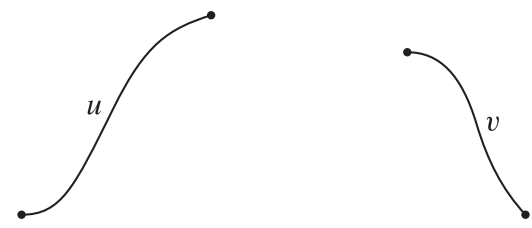

Fig. 10

Suppose that

$$
\text { whenever } u(t)=v(s) \text { for some } \alpha<s<\beta \text { we have } \ddot{u}(t) \leq v^{\prime \prime}(s) \text {. }
$$

Then $v$ is a reflection of $u: v(t) \equiv u(c-t)$ where $c=b+\alpha=a+\beta$. In particular, $v(\alpha)=u(b)$.

Lemma 4.1 is equivalent to

Lemma 4.1. In the hypotheses of Lemma 4.1, if we change (33) and 34, to

$$
\dot{u}(b)=0
$$

and

and change (35) to

$$
\dot{u}<0 \quad \text { on }(a, b)
$$

$$
v(\alpha) \leq u(a), \quad v(\beta)=u(b), \quad v(\beta)<v(s)<v(\alpha), \quad \forall \alpha<s<\beta,
$$

then $v$ is a translate of $u$.

Proof of the equivalence of Lemma 4.1 and Lemma 4.1. Let $U(t)=u(-t)$ and $V(t)=$ $v(t)$. 
Proof of Lemma 4.1. Reflect $v$ about $\alpha$, i.e. set

$$
w(t):=v(2 \alpha-t), \quad 2 \alpha-\beta<t<\alpha .
$$

Shift $w$ far to the right and then slide it to the left until the graph first touches that of $u$. If the touching occurs at $(c, u(c))$ for some $a<c<b$, then, by Lemma 2.1, the shift of $w$ coincides with $u$ near $c$, which in turn implies that they coincide everywhere and $v$ is a reflection of $u$ as desired. Since $\dot{w}(\beta)=\dot{v}(\alpha)=0$ while $\dot{u}>0$ on $(a, b)$, there are only two possibilities: The above situation does not occur but the touching occurs at $(a, 0)$ or at $(b, u(b))$. If the touching occurs at $(a, 0)$, then we must have $\dot{v}(\beta)=0$ since $\dot{u}(a)=0$. By Theorem 2.1 and Lemma 2.1. the two graphs must be identical near the origin. Impossible. If the touching occurs at $(b, u(b))$, then we must have $\dot{u}(b)=0$ since we know that $\dot{v}(\alpha)=0$. Let $\bar{w}$ denote the shift. We know that for some $\epsilon>0$,

$$
\bar{w}(b)=v(\alpha)=u(b), \quad \bar{w}<u(b) \quad \text { on }(b-\epsilon, b) .
$$

Turning the picture upside down, and applying Theorem 2.1, we again get a contradiction. More precisely, let

$$
U(t):=\bar{w}(t)-\bar{w}(b-t), \quad V(t)=u(b)-u(b-t), \quad 0<t<\epsilon .
$$

Applying Theorem 2.1 to $U$ and $V$ leads to $U \equiv V$ near the origin, i.e. $u \equiv \bar{w}$ near $b$. Impossible. Lemma 4.1 is established.

Lemma 4.1 is also equivalent to

Lemma 4.1 $]^{\prime}$. Let $u \in C^{2}((a, b)) \cap C^{1}([a, b])$ satisfy

$$
\dot{u}(b)=0, \quad u(a)>u(t)>u(b) \quad \text { for } a<t<b,
$$

and let $v \in C^{2}((a, b)) \cap C^{1}([\alpha, \beta])$ satisfy

$$
\dot{v}(\beta)=0, \quad \dot{v}>0,
$$

and

$$
v(\alpha) \leq u(b), \quad v(\beta)=u(a) .
$$

Finally, assume that

$$
\text { whenever } u(t)=v(s) \text { we have } \ddot{u}(t) \leq v^{\prime \prime}(s) \text {. }
$$

Then $v$ is a reflection of $u$ and $v(\alpha)=u(b)$.

Proof of the equivalence of Lemma 4.1 and Lemma 4.1$]^{\prime}$. Let $V(t)=-u(-t)$ and $U(t)=$ $-v(-t)$.

Theorem 4.1. Let $u \in C^{2}((0, b)) \cap C^{1}([0, b])$ be positive on $(0, b)$ and satisfy

$$
u(0)=\dot{u}(0)=u(b)=0 .
$$

Let $a$ be the first point where $u$ achieves its maximum and assume that

$$
\dot{u}>0 \quad \text { on }(0, a) \text {. }
$$


Assume furthermore (main condition) that

$$
\text { whenever } u(t)=u(s) \text { for } t<s \text { we have } \ddot{u}(t) \leq u^{\prime \prime}(s) .
$$

Then $u$ is symmetric about $b / 2$ and

$$
u \equiv u(a) \quad \text { on }[a, b-a] .
$$

Note that we do not assume that $\dot{u}(b)=0$.

Remark 4.1. The condition $\dot{u}(0)=0$ cannot be dropped. Indeed, we could consider a positive symmetric function on some interval $(0, b)$ satisfying 37 except that $\dot{u}(0)>0$. Then, near $b$ we could change $u$ slightly by increasing its second derivative there, in such a way that the new function, when extended, would still vanish at some point $\bar{b}>b$. The resulting function on $(0, \bar{b})$ would satisfy $\sqrt{38}$ but would not be symmetric.

Here is an example showing that if condition $(38)$ is weakened to $\dot{u} \geq 0$ on $(0, a)$, then $u$ need not be symmetric. Here $u$ on $(4,5)$ is the reflection of $u$ on $(0,1)$.

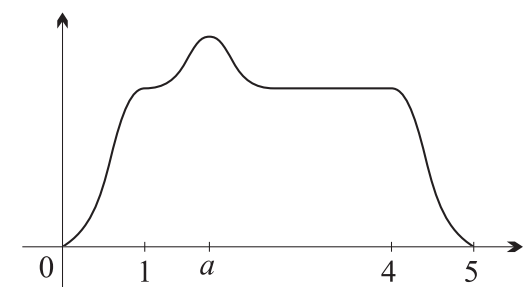

Fig. 11

Proof of Theorem 4.1 Let $b_{1}$ be the last value of $t$ where $u$ assumes its maximum. By Lemma 4.1, $b_{1}=b-a$ and

$$
u(t)=u(b-t) \quad \text { for } 0 \leq t \leq a .
$$

Now we prove that $u$ is constant on $\left[a, b_{1}\right]$. If not, we can find $[\alpha, \beta] \subset\left[a, b_{1}\right]$ such that

$$
u(a) \geq u(\alpha)>u(t)>u(\beta)=\min _{\left[a, b_{1}\right]} u>0 \quad \text { for } \alpha<t<\beta .
$$

Since $u(t)=u(b-t)$ on $(0, a)$, it follows from the main condition that if $t \leq a<s$ and $u(t)=u(s)$ then $\ddot{u}(t)=u^{\prime \prime}(s)$. Thus for $s$ in $(\alpha, \beta)$ we can find a unique $t(s)$ in $(0, a)$ such that

$$
u(t(s))=u(s)
$$

Hence

$$
\dot{u}(t(s)) t^{\prime}=u^{\prime}(s)
$$

and

$$
\left(\dot{u}(t(s))^{2}-u^{\prime}(s)^{2}\right)^{\prime}=2 u^{\prime}(s)\left(\ddot{u}(t(s))-u^{\prime \prime}(s)\right)=0 .
$$

Hence $\dot{u}(t(s))^{2}=u^{\prime}(s)^{2}$. This is impossible, since $u^{\prime}(\beta)=0$, while $\dot{u}(t(\beta))>0$. Theorem 4.1 is proved. 


\section{General second order operators}

In this section we extend various results to nonlinear second order ordinary differential equations. We consider

$$
K \in C^{0}\left(\mathbb{R}^{3}\right), \quad K(s, p, q) \text { is } C^{1} \text { in }(p, q), \quad K_{q}(s, p, q)>0, \forall(s, p, q) \in \mathbb{R}^{3},
$$

and we study the nonlinear second order differential operator $K(u, \dot{u}, \ddot{u})$. It is elliptic because of (41).

The first result is an extension of Lemma 2.1.

Lemma 5.1. Let $K$ satisfy 41 , and let $u \geq v$ be two $C^{2}$ functions on $(0, b)$ satisfying

$$
\max \{\dot{u}, \dot{v}\}>0 \quad \text { on }(0, b),
$$

and

$$
\text { if } u(t)=v(s) \text { for } 0<t<s<b \text { then } K(u(t), \dot{u}(t), \ddot{u}(t)) \leq K\left(v(s), v^{\prime}(s), v^{\prime \prime}(s)\right) \text {. }
$$

Then either

$$
u>v \quad \text { on }(0, b)
$$

or

$$
u \equiv v \quad \text { on }(0, b)
$$

Proof. Suppose $u(c)=v(c)$ for some $0<c<b$. Then, by 42, $\dot{u}(c)=\dot{v}(c)>0$. By the implicit function theorem, for $s$ close to $c$, there is a $C^{2}$ function $t(s)$ such that

$$
u(t(s))=v(s)
$$

Set, for $s$ close to $c$,

$$
g(s)=s-t(s),
$$

so $g \geq 0$. Differentiating (45) we find

$$
\dot{u}(t(s)) t^{\prime}=v^{\prime}(s), \quad \ddot{u} t^{\prime 2}+\dot{u} t^{\prime \prime}=v^{\prime \prime} .
$$

Thus, for some functions $c_{1}(s)$ and $c_{2}(s)$, we have

$$
\dot{u}(t(s))-v^{\prime}(s)=c_{1}(s) g^{\prime}(s), \quad \ddot{u}(t(s))-v^{\prime \prime}(s)=\dot{u} g^{\prime \prime}+c_{2}(s) g^{\prime} .
$$

Using (41) and the above, we obtain, via the mean value theorem,

$$
0 \geq K(u, \dot{u}, \ddot{u})-K\left(v, v^{\prime}, v^{\prime \prime}\right)=a(s) \dot{u} g^{\prime \prime}+c(s) g^{\prime} \quad \text { with } a(s)>0 .
$$

Now if $u(s)=v(s)$ for some $s>0$ then $g$ vanishes there. The strong maximum principle applied to (12) implies that $v \equiv u$ in a neighborhood of $c$, and 44 follows immediately. Lemma 5.1 is established.

The second result is an extension of Lemma 2.4 
Lemma 5.2. Let $K$ satisfy 441 , and let $u, v \in C^{2}((0, b)) \cap C^{0}([0, b])$ satisfy

$$
\begin{gathered}
\quad v(0) \leq u(0), \quad v(b) \leq u(b), \quad v<u(b) \text { on }(a, b), \\
\text { either } \quad \dot{u}>0 \quad \text { on } \quad(0, b) \text { or } \dot{v}>0 \text { whenever } u(0)<v<u(b),
\end{gathered}
$$

and 43 . Then

$$
u \geq v \quad \text { on }[0, b] .
$$

Proof. The proof is essentially the same as that of Lemma 2.4 The only difference is that we use Lemma 5.1 instead of Lemma 2.1.

The third result is an extension of Lemma 2.3

Lemma 5.3. Let $u$ and $w$ be positive $C^{1,1}$ functions in $(0, c)$, belonging to $C^{1}([0, c))$, and both satisfying

$$
\frac{d}{d t} K(\dot{u})=f(u)
$$

and

$$
u(0)=w(0)=0, \quad \dot{u}(0)=\dot{w}(0) .
$$

Here

$$
K \in C^{1}(\mathbb{R}), \quad K^{\prime}>0 \quad \text { in } \mathbb{R} .
$$

Assume in addition that $K^{\prime}$ is even, and assume that $f(\varrho)$ is locally Lipschitz for $\varrho>0$ (not necessarily for $\varrho \geq 0$ ). Then

$$
u \equiv w .
$$

Proof. The proof is similar to that of Lemma 2.3. Multiplying (47) by $\dot{u}$ we find that

$$
\frac{d}{d t} G(\dot{u})=\frac{d}{d t} F(u)
$$

where

$$
G(p)=\int_{0}^{p} \varrho K^{\prime}(\varrho) d \varrho \quad \text { and } \quad F \text { is such that } F_{u}=f(u) .
$$

Integrating this from $t_{0}$ to $t, t_{0}>0$, we find

$$
G(\dot{u}(t))-G\left(\dot{u}\left(t_{0}\right)\right)=F(u(t))-F\left(u\left(t_{0}\right)\right) .
$$

Letting $t_{0} \rightarrow 0$ we see that $F(u)$ has a limit at $u=0$, which we may take to be zero. Thus

$$
G(\dot{u})=F(u) .
$$

As in the proof of Lemma 2.3, we have $\dot{u}>0$ on $(0, c / 2)$. Since $K^{\prime}>0$, we see that

$$
G(p)>0, \quad G^{\prime}(p)>0 \quad \text { for } p>0 .
$$

Thus

$$
F(u(t))>0 \quad \text { for } 0<t<c / 2 .
$$


It follows that

$$
\frac{\dot{u}}{G^{-1}(F(u(t))}=1 \quad \text { on }(0, c / 2) .
$$

If, on $\varrho>0, H(\varrho)$ is such that

$$
H_{\varrho}=\frac{1}{G^{-1}(F(\varrho))},
$$

we find that

$$
\frac{d}{d t} H(u)=1 .
$$

Integrating from $t_{0}>0$ to $t>t_{0}$ we obtain

$$
H(u(t))-H\left(u\left(t_{0}\right)\right)=t-t_{0} .
$$

Letting again $t_{0} \rightarrow 0$ we see that $H$ has a limit at $\varrho=0$, which we may take to be zero. Thus

$$
H(u(t))=t .
$$

So $u(t)$ is uniquely determined on $(0, c / 2)$, since $H_{\varrho}>0$ for $\varrho>0$. Then, by the local Lipschitz property of $f(\varrho)$ on $\varrho>0$, it follows that $u$ is unique on $(0, c)$. Lemma 5.3 is proved.

The fourth result is an extension of Lemma 2.2. Here we do not assume that $K^{\prime}$ is even.

Lemma 5.4. Let $K$ satisfy 48, and let $u$ and $w$ be positive $C^{1,1}$ functions on $(0, c)$, belonging to $C^{1}([0, c))$, and satisfying, for some $f \in L^{\infty}(0, \infty)$,

$$
\frac{d}{d t} K(\dot{u})=f(u), \quad \frac{d}{d t} K(\dot{w})=f(w) \quad \text { on }(0, c),
$$

and

$$
u(0)=w(0)=0, \quad \dot{u}(0)=\dot{w}(0), \quad \dot{u}>0 \text { on }(0, c) .
$$

Then

$$
u \equiv w \quad \text { on }(0, c) .
$$

Proof. Follow the proof of Lemma 5.3 until 52. In a similar way we also have

$$
G(\dot{w}(t))=F(w(t)) \quad \text { on }(0, c) .
$$

Let $0<b<c$ be any number satisfying (17). We only need to prove (18).

By (52), (54) and the fact that $\dot{u}>0$ on $(0, c)$, we know that

$$
G(\dot{w}(t))=F(w(t))>0, \quad \forall 0<t<b .
$$

Since $w(0)=0$ and $w>0$ on $(0, b)$, we have $\dot{w}>0$ on $(0, b)$. Proceeding as in the proof of Lemma 5.3 , we arrive at

$$
H(u(t))=H(w(t))=t, \quad 0<t<b .
$$

But $H_{\varrho}(\varrho)>0$ for $\varrho>0$ and $H(0)=0$, so we obtain (18). Lemma 5.4 is proved.

The fifth result is an extension of Lemma 3.1 . 
Lemma 5.5. Let $K$ satisfy [48], and let, for some $b>0, u, v \in C^{2}((0, b)) \cap C^{1}([0, b])$ satisfy

$$
u(t) \geq v(t)>0 \quad \text { for } 0<t \leq b,
$$

either $\dot{u}(t)>0, \dot{v}(t) \geq 0$ for $0<t \leq b, \quad$ or $\quad \dot{u}(t) \geq 0, \dot{v}(t)>0$ for $0<t \leq b$,

and

$$
u(0)=\dot{u}(0)=0 .
$$

Assume

$$
\text { whenever } u(t)=v(s) \text { for } 0<t<s<b \quad \text { we have } \quad \frac{d}{d t} K(\dot{u}(t)) \leq \frac{d}{d s} K(\dot{v}(s)) \text {. }
$$

Then

$$
u \equiv v \quad \text { on }[0, b]
$$

Proof. The proof is similar to that of Lemma 3.1. We will only prove it under the assumption " $\dot{u}(t)>0, \dot{v}(t) \geq 0$ for $0<t \leq b$ ", since the changes needed when assuming instead " $\dot{u}(t) \geq 0, \dot{v}(t)>0$ for $0<t \leq b$ " are similar to those in the proof of Theorem 2.1 We start as in the proof of Theorem 2.1] $u$ satisfies (47) for some unknown continuous function $f$ on $[0, u(b)]$.

Condition (55) means

$$
\left.K^{\prime}(\dot{v}(s))\right) \ddot{v} \geq f(v) .
$$

Multiply (47) by $\dot{u}$; we find (49) with $G$ given by (50). (52) still holds, so does (53).

Multiplying (57) by $\dot{v} \geq 0$ we obtain

$$
\frac{d}{d t} G(\dot{v}) \geq \frac{d}{d t} F(v) \quad \text { for } 0<t_{0}<t .
$$

Since 53) still holds, and since $\dot{v} \geq 0$, we have

$$
G(\dot{v}(t)) \geq 0 \quad \text { for } 0 \leq t \leq b .
$$

Thus, also in view of our choice of setting $F(0)=0$, we find by integrating

$$
G(\dot{v}) \geq F(v) .
$$

Because of the second inequality in (53), $(52)$ and $(58)$ imply $(32)$, and the rest of the proof follows exactly as the arguments after 323 in the proof of Lemma 3.1 Lemma 5.5 is established.

The sixth result is closely related to Lemma 4.1 .

Lemma 5.6. Let $K$ satisfy (48), and let $u \in C^{2}((a, b)) \cap C^{1}([a, b])$ satisfy $\left.\sqrt{33}\right)$ and 34 . Let $v \in C^{2}((\alpha, \beta)) \cap C^{1}([\alpha, \beta])$ satisfy $[35$, , 36) and

$$
\dot{v} \leq 0 \quad \text { on }(\alpha, \beta) \text {. }
$$




\section{Suppose that}

$$
\text { whenever } \left.u(t)=v(s) \text { for some } \alpha<s<\beta \quad \text { we have } \frac{d}{d t} K(\dot{u}(t)) \leq \frac{d}{d s} K(\dot{v}(s))\right) \text {. }
$$

Then $v$ is a reflection of $u: v(t) \equiv u(c-t)$ where $c=b+\alpha=a+\beta$. In particular, $v(\alpha)=u(b)$.

Remark 5.1. There are analogues of Lemmas 4.1, 4.1]', which we call Lemmas 5.6, 5.6', and which follow from Lemma 5.6 as do the former from Lemma 4.1

Proof of Lemma 5.6. Follow the proof of Lemma 4.1 and make the following changes: Change "by Lemma 2.1' to "by Lemma 5.1]'; change " by Theorem 2.1 and Lemma 2.1, to "by Lemma 5.5]'; change "applying Theorem 2.1]' to "applying Lemma 5.5.'

Question 5.1. Do the conclusions of Lemmas 5.3, 5.4 5.5 and 5.6 still hold if we replace $\frac{d}{d t} K(\dot{u}(t)) \leq \frac{d}{d s} K(\dot{v}(s))$ in 55 by $K(u(t), \dot{u}(t), \ddot{u}(t)) \leq K\left(v(s), v^{\prime}(s), v^{\prime \prime}(s)\right)$ for some $K$ satisfying (41), or even for those $K(s, p, q)$ which are independent of $s$ ?

\section{Counter-examples}

We will give an example showing that the answer to Question 1.2 is no.

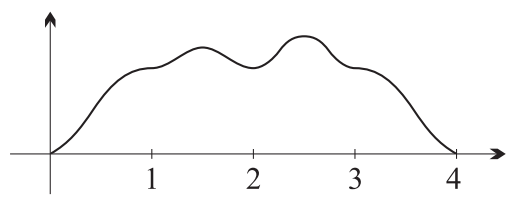

Fig. 12

First, in Fig. 12 we present a function $u$ on $(0,4)$. Here, $u$ on $(3,4)$ is the reflection of its values on $(0,1), u$ will satisfy the curvature condition:

$$
\frac{\ddot{u}(t)}{\left(1+\dot{u}(t)^{2}\right)^{3 / 2}} \leq \frac{u^{\prime \prime}(s)}{\left(1+u^{\prime}(s)^{2}\right)^{3 / 2}} \quad \text { for any } t<s \text { such that } u(t)=u(s) .
$$

$u$ will be taken to be symmetric on $(1,2)$ about $3 / 2$, and symmetric on $(2,3)$ about $5 / 2$. We will then require 60 only for $1 \leq t \leq 3 / 2$ and $2 \leq s \leq 5 / 2$.

For convenience, after subtracting a constant, and shifting, we may describe the two bumps by two functions $u$ and $v$ (we still call the first $u$ ) on $(0,1)$ given by

$$
u=\epsilon^{6} t^{3}(1-t)^{3}, \quad v=\epsilon^{3} t^{3}(1-t)^{3}
$$

with $\epsilon$ very small.

Claim. Whenever $u(t)=v(s)$ for $t<1 / 2$, then

$$
\frac{\ddot{u}(t)}{\left(1+\dot{u}(t)^{2}\right)^{3 / 2}} \leq \frac{v^{\prime \prime}(s)}{\left(1+v^{\prime}(s)^{2}\right)^{3 / 2}} \text {. }
$$


Proof. $u(t)=v(s)$ means

$$
\epsilon t(1-t)=s(1-s) .
$$

We only have to check that $u$ and $v$ in (61) satisfy

$$
\text { if } u(t)=v(s) \text { for } 0<t, s<1 / 2 \text { then } \frac{\ddot{u}(t)}{\left(1+\dot{u}(t)^{2}\right)^{3 / 2}} \leq \frac{v^{\prime \prime}(s)}{\left(1+v^{\prime}(s)^{2}\right)^{3 / 2}} .
$$

Indeed, from 62, we see that for $\epsilon$ small,

$$
s=\epsilon\left(t-t^{2}\right)+O\left(\epsilon^{2}\left(t-t^{2}\right)^{2}\right) .
$$

Now

$$
\begin{aligned}
& \dot{u}=\epsilon^{6} 3(1-2 t)\left(t-t^{2}\right)^{2}, \\
& \ddot{u}=6 \epsilon^{6}\left[-\left(t-t^{2}\right)^{2}+(1-2 t)^{2}\left(t-t^{2}\right)\right]=6 \epsilon^{6}\left(t-t^{2}\right)\left(1-5 t+5 t^{2}\right) .
\end{aligned}
$$

Thus

At the same time

$$
\frac{\ddot{u}}{\left(1+\dot{u}^{2}\right)^{3 / 2}} \leq C \epsilon^{6}\left(t-t^{2}\right)
$$

$$
\frac{v^{\prime \prime}}{\left(1+\left(v^{\prime}\right)^{2}\right)^{3 / 2}}=\epsilon^{3}\left[6 s+O\left(s^{2}\right)\right] \geq 5 \epsilon^{4}\left(t-t^{2}\right),
$$

by (64). It follows that 63 holds for $\epsilon$ small.

Finally, we obtain an example of a closed nonconvex curve $M$ satisfying $H(A) \leq H(B)$ if $A, B$ lie on $M$ and $A_{1} \leq B_{1}$, but with $H(A)$ not equal to $H(B)$.

Namely take the curve $u$ above and round it off on the bottom in a symmetric way.

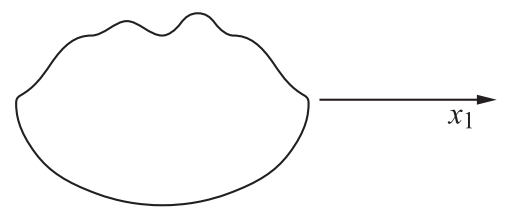

Fig. 13

\section{Open problems in higher dimensions}

The problems are related to Theorem 2.1 and to Lemmas 2.2 and 2.3. For convenience, we will denote the independent variables by $(t, y), t$ nonnegative, $y$ in $\mathbb{R}^{n-1}$. The functions we consider are defined in the closure of the half-ball

$$
B^{+}:=\left\{\left.(t, y)\left|t^{2}+\right| y\right|^{2}<R^{2}, t>0\right\} .
$$

The first question is related to Theorem 2.1 
Problem 7.1. Suppose $u \geq v>0$ and $u_{t}:=\partial u / \partial t>0$ in $B^{+}$, and $u$ and $v$ are $C^{2}$ in the closure of $B^{+}$. Suppose

$$
u=v=u_{t}=v_{t}=0 \quad \text { on }\{(0, y)|| y \mid<R\} .
$$

Assume the main condition:

$$
\text { whenever } u(t, y)=v(s, y) \text { for } t<s, \quad \text { then } \quad \Delta u(t, y) \leq \Delta v(t, y) .
$$

Question: Is $u \equiv v$ ?

Problem 7.2. Let $u$ and $v$ be $C^{\infty}$ in the closure of $B^{+}$and positive in $B^{+}$, and satisfy $\Delta u=f(y, u), \Delta v=f(y, v)$ and 677 . Assume that $f$ is continuous in $u \geq 0$, smooth in $y$ there, and smooth in $(y, u)$ where $u>0$. Question: Is $u \equiv v$ ?

In Part II we prove the answer is yes, but under the additional assumptions that $u$ and $v$ vanish of finite order in $t$ at the origin and that $u \geq v$.

Problem 7.3. Is the answer to Problem 7.2 yes if we add the hypothesis that $u_{t}>0$ when $t>0$ ?

\section{References}

[1] Aleksandrov, A. D.: Uniqueness theorems for surfaces in the large V. Vestnik Leningrad. Univ. 13, no. 19, 5-8 (1958) (in Russian) MR 0102114

[2] Li, Y.Y.: Group invariant convex hypersurfaces with prescribed Gauss-Kronecker curvature. in: Contemp. Math. 205, Amer. Math. Soc., 203-218 (1997) Zbl 0881.53051 MR 1447225

[3] Li, Y.Y., Nirenberg, L.: A geometric problem and the Hopf Lemma. II. Chinese Ann. Math. Ser. B 27, 193-218 (2006)

[4] Mawhin, J.: Points fixes, points critiques et problèmes aux limites. Sém. Math. Sup. 92, Presses Univ. Montréal, Montreal, QC (1985) Zbl 0561.34001 MR 0789982

[5] Ros, A.: Compact hypersurfaces with constant higher order mean curvatures. Rev. Mat. Iberoamericana 3, 447-453 (1987) Zbl 0673.53003 MR 0996826

[6] Wente, H. C.: Counterexample to a conjecture of H. Hopf. Pacific J. Math. 121, 193-243 (1986) Zbl 0586.53003 MR 0815044 